\title{
BOUNDARY MODULUS OF CONTINUITY AND QUASICONFORMAL MAPPINGS
}

\author{
Miloš Arsenović, Vesna Manojlović and Raimo Näkki \\ University of Belgrade, Faculty of Mathematics \\ Studentski Trg 16, 11000 Belgrade, Serbia; arsenovic@matf.bg.ac.rs \\ University of Belgrade, Faculty of Organizational Sciences \\ Jove Ilića 154, 11000 Belgrade, Serbia; vesnam@fon.bg.ac.rs \\ University of Jyväskylä, Department of Mathematics and Statistics \\ P.O. Box 35 (MaD), FI-40014 Jyväskylä, Finland; raimon@maths.jyu.fi
}

\begin{abstract}
Let $D$ be a bounded domain in $\mathbf{R}^{n}, n \geq 2$, and let $f$ be a continuous mapping of $\bar{D}$ into $\mathbf{R}^{n}$ which is quasiconformal in $D$. Suppose that $|f(x)-f(y)| \leq \omega(|x-y|)$ for all $x$ and $y$ in $\partial D$, where $\omega$ is a non-negative non-decreasing function satisfying $\omega(2 t) \leq 2 \omega(t)$ for $t \geq 0$. We prove, with an additional growth condition on $\omega$, that $|f(x)-f(y)| \leq C \max \left\{\omega(|x-y|),|x-y|^{\alpha}\right\}$ for all $x, y \in D$, where $\alpha=K_{I}(f)^{1 /(1-n)}$.
\end{abstract}

\section{Introduction}

The following result, due to Martio and one of the authors of this article, was established in $[\mathrm{MN}]$ :

If $D$ is a bounded domain in $\mathbf{R}^{n}, n \geq 2$, and if $f$ is a continuous mapping of $\bar{D}$ into $\mathbf{R}^{n}$ which is quasiconformal in $D$ and satisfies

$$
|f(x)-f(y)| \leq M|x-y|^{\alpha}
$$

for some $M>0,0<\alpha \leq 1$, and for all $x$ and $y$ in $\partial D$, then

$$
|f(x)-f(y)| \leq M^{*}|x-y|^{\beta}
$$

for all $x$ and $y$ in $D$, where $\beta=\min \left\{\alpha, K_{I}(f)^{1 /(1-n)}\right\}$ and $M^{*}$ depends only on $M$, $\alpha, n, K(f)$ and $\operatorname{diam}(D)$.

Our goal in the present paper is to extend this theorem so as to allow in (1.1) majorizing conditions that are more general than a Hölder condition. For this purpose, and in accordance with the terminology used by Hinkkanen in [H1] and [H2], we call a non-negative non-decreasing function $\omega$, defined for $t \geq 0$, a majorant if

$$
\omega(2 t) \leq 2 \omega(t)
$$

for all $t \geq 0$. For example, if $\omega(t)=M t^{\alpha}$, where $M>0$ and $0 \leq \alpha \leq 1$, then $\omega$ is a majorant. More generally, if $\omega$ is subadditive, as is often the case in moduli of continuity considerations, that is, if $\omega\left(t_{1}+t_{2}\right) \leq \omega\left(t_{1}\right)+\omega\left(t_{2}\right)$ for $t_{1}, t_{2} \geq 0$, then $\omega$ is

doi:10.5186/aasfm.2012.3718

2010 Mathematics Subject Classification: Primary 30C65.

Key words: Quasiconformal mapping, modulus of continuity.

The first two authors were supported by the Ministry of Science, Serbia, projects OI174017 and OI174024. 
a majorant. We remark that $\omega$ need not be continuous, that we may have $\omega(0)>0$ and that

$$
\omega(A t) \leq 2 A \omega(t)
$$

for all $t \geq 0$ and $A \geq 1$.

We will replace condition (1.1) by

$$
|f(x)-f(y)| \leq \omega(|x-y|),
$$

where $\omega$ is a majorant subject to an additional growth condition (see Theorem 3.1 in Section 3). To wit, we assume that on the boundary of $D$ the modulus of continuity of $f$ is majorized by such a function $\omega$. Our conclusion then will be that

$$
|f(x)-f(y)| \leq C \hat{\omega}(|x-y|)
$$

for all $x$ and $y$ in $D$, where $C>0$ is a constant and

$$
\hat{\omega}(t)=\max \left\{\omega(t), t^{\alpha}\right\}
$$

with $\alpha=K_{I}(f)^{1 /(1-n)}$. No constraint in regard to the regularity of the boundaries of $D$ and $f(D)$ will be imposed. Much attention has been lavished on the case where the domains are smooth. See, for example, $[\mathrm{K}],[\mathrm{KM}]$ and the references therein. Our argument for establishing the generalization indicated above closely parallels the pattern presented in [MN]. In Section 3 we extend, furthermore, a related result in [HN, Theorem 4], concerning mappings of a ball, to a wider range of domains, that is, to domains with uniformly perfect boundaries.

In matters regarding notation and terminology we will conform to the usage in the book of Väisälä [V]. In particular, $K_{I}(f), K_{O}(f)$ and $K(f)$ will signify the inner, the outer and the maximal dilatations of a quasiconformal mapping $f$, respectively. The conformal modulus of a curve family $\Gamma$ is designated by $M(\Gamma)$. Given sets $E, F$ and $G$, we denote by $\Delta(E, F ; G)$ the family of all curves in $G$ joining $E$ to $F$. Unless otherwise stipulated, all sets considered will lie in the euclidean $n$-space $\mathbf{R}^{n}, n \geq 2$.

Acknowledgement. The authors wish to acknowledge the suggestions offered to them by conversations with Aimo Hinkkanen.

\section{Preliminary considerations}

For the convenience of the readers of this article we assemble in this section a number of results from $[\mathrm{MN}]$, appropriately adapted and generalized to accommodate our present needs. We commence with a most elementary observation:

Lemma 2.1. Let $D$ be a proper subdomain of $\mathbf{R}^{n}$ and let $f$ be a mapping defined in $\bar{D}$. Suppose that for some majorant $\omega$,

$$
|f(y)-f(z)| \leq \omega(|y-z|)
$$

whenever either $y, z \in \partial D$ or $y \in D$ and $z$ is a point in $\partial D$ closest to $y$. Then

$$
|f(x)-f(y)| \leq 3 \omega(|x-y|)
$$

for all $x \in \partial D, y \in D$. 
Proof. Fix $x \in \partial D$ and $y \in D$. Let $z$ be a point in $\partial D$ closest to $y$. Since $|z-y| \leq|x-y|$ and $|x-z| \leq 2|x-y|$, condition (1.2) yields

$$
\begin{aligned}
|f(x)-f(y)| & \leq|f(x)-f(z)|+|f(z)-f(y)| \\
& \leq \omega(2|x-y|)+\omega(|x-y|) \leq 3 \omega(|x-y|) .
\end{aligned}
$$

In our investigation on the modulus of continuity we will resort to two separate strategies which hinge upon the capacity density of the boundary. The thick portions of the boundary will be handled by employing standard extremal length techniques:

Lemma 2.2. Let $y$ be a point in a domain $D$, let $x$ be a point in $\partial D$ closest to $y$, set $d=|x-y|$ and let $m>0$. Suppose that

$$
\operatorname{cap}\left(\bar{B}\left(y, \frac{1}{2} d\right), D\right) \geq m \text {. }
$$

If $f$ is a continuous mapping of $\bar{D}$ into $\mathbf{R}^{n}$ which is quasiconformal in $D$ and if

$$
|f(x)-f(z)| \leq \omega(|x-z|)
$$

for all $z \in \partial D$ and for some majorant $\omega$, then

$$
|f(x)-f(y)| \leq C \omega(|x-y|),
$$

where $C>0$ is a constant depending only on $m, n$ and $K(f)$.

Proof. We emulate the proof of Lemma 8 in $[\mathrm{MN}]$ and present only the necessary adjustments needed to deal with $\omega(t)$ instead of powers of $t$.

Fix an integer $p>2$ such that

$$
\frac{\omega_{n-1}}{[\log 2(p-1)]^{n-1}} \leq \frac{m}{2},
$$

where $\omega_{n-1}$ denotes the $(n-1)$-dimensional surface area of the unit sphere. Set $B=B(y, d / 2), B^{\prime}=f(B), d^{\prime}=d\left(f(x), \partial B^{\prime}\right)$. We begin by showing that

$$
d^{\prime} \leq M_{0} \omega(d),
$$

where $M_{0}=2 p \exp \left(\left[2 K_{O}(f) \omega_{n-1} / m\right]^{1 /(n-1)}\right)$.

Since (2.3) holds trivially if $d^{\prime} \leq p \omega(d)$, we may assume that $d^{\prime}>p \omega(d)$. Consider the curve family $\Gamma=\Delta(B, \partial D \cap B(x, p d) ; D)$. The minorizing principle for the modulus, together with (2.1), implies that

$$
M(\Gamma) \geq \frac{m}{2} .
$$

See [MN, p. 347]. Thus, by the quasiconformality of $f$,

$$
M(f \Gamma) \geq \frac{m}{2 K_{O}(f)} .
$$

On the other hand, our hypothesis on the boundary distortion at $x$ guarantees that the set $f(\partial D \cap B(x, p d))$ is contained in $B(f(x), \omega(p d))$. Hence the curve family $f \Gamma$ is minorized by the family $\Delta\left(S(f(x), \omega(p d)), S\left(f(x), d^{\prime}\right) ; \mathbf{R}^{n}\right)$, and therefore

$$
M(f \Gamma) \leq \omega_{n-1}\left(\log \frac{d^{\prime}}{\omega(p d)}\right)^{1-n} .
$$

In conjunction with (2.4) this leads to

$$
d^{\prime} \leq \exp \left(\left[2 K_{0}(f) \omega_{n-1} / m\right]^{1 /(n-1)}\right) \omega(p d),
$$

and (2.3) ensues because $\omega(p d) \leq 2 p \omega(d)$ by (1.3). 
To complete the proof, we can repeat the argument in [MN, p. 348] verbatim to produce the estimate

$$
|f(x)-f(y)| \leq \exp \left(\frac{K_{I}(f) \omega_{n-1}}{b_{n}(\log 2)^{n-1}}\right) d^{\prime}
$$

where $b_{n}>0$ is a cap-inequally constant depending only on $n$. See [V, 10.2]. Invoking (2.3) this yields $(2.2)$ with $C=M_{0} \exp \left(\frac{K_{I}(f) \omega_{n-1}}{b_{n}(\log 2)^{n-1}}\right)$.

We next focus our attention on the thin parts of the boundary. Three lemmas will be acquired. The first of these seeks to describe a certain quasisymmetry property of a quasiconformal mapping at a boundary point. The lemma is extracted directly from [MN, p. 342]. We put to use standard notation

$$
\begin{gathered}
L(r)=L(x, f, r)=\max _{|y-x|=r}|f(x)-f(y)|, \\
l(r)=l(x, f, r)=\min _{|y-x|=r}|f(x)-f(y)|
\end{gathered}
$$

for a mapping $f$ defined and continuous in $\{x\} \cup S(x, r)$.

Lemma 2.3. Let $y$ be a point in a bounded domain $D$, let $x$ be a point in $\partial D$ closest to $y$ and set $d=|x-y|$. Suppose that

$$
\operatorname{cap}(\bar{B}(y, d / 2), D)<2^{-(n+3)} b_{n} .
$$

If $\mathrm{f}$ is a continuous mapping of $\bar{D}$ into $\mathbf{R}^{n}$ which is quasiconformal in $D$ and if the boundary component of $D$ whose image under $f$ separates $f(D)$ from the point $\infty$ in $\overline{\mathbf{R}}^{n}$ is not contained in $B(x, 4 d)$, then $D$ contains a sphere $S(x, r), d<r<2 d$, on which

$$
\frac{L(r)}{l(r)} \leq c
$$

where $c \geq 1$ depends only on $n$ and $K_{O}(f)$.

Lemma 2.4. Let $r>0$, let $0<a<1<b$ and let $x$ be a boundary point of a bounded domain $D$ containing the closed spherical ring $\bar{B}(x, b r) \backslash B(x, a r)$. Suppose that $f$ is a continuous mapping of $\bar{D}$ into $\mathbf{R}^{n}$ which is quasiconformal in $D$ and that

$$
|f(x)-f(z)| \leq \omega(|x-z|)
$$

for all $z \in \partial D$, where $\omega$ is a majorant satisfying

$$
\frac{\omega(t)}{t^{\alpha}} \leq M \max \left\{1, \frac{\omega(s)}{s^{\alpha}}\right\}
$$

for some $M>0$, for $\alpha=K_{I}(f)^{1 /(1-n)}$ and for all $0<s<t<\operatorname{diam}(D)$. Then

$$
|f(x)-f(y)| \leq C \max \left\{\omega(|x-y|),|x-y|^{\alpha}\right\}
$$

for all $y \in S(x, r)$, where $C$ depends only on $a, b, n, M$ and $K_{O}(f)$. In particular, if $\omega(t) / t^{\alpha}$ is decreasing for $0<t<\operatorname{diam}(D)$, then

$$
|f(x)-f(y)| \leq \hat{C} \omega(|x-y|)
$$

for all $y \in S(x, r)$, where $\hat{C}$ depends only on $a, b, n$ and $K_{O}(f)$. 
Proof. If $x$ is mapped by $f$ into the unbounded component of $\mathbf{R}^{n} \backslash f(S(x, r))$, then, since $f(\bar{D})$ is bounded, $B(x, r)$ contains a boundary component $E$ of $D$ such that $f(E)$ separates $f(D)$ from the point $\infty$ in $\overline{\mathbf{R}^{n}}$. Accordingly

$$
\begin{aligned}
|f(x)-f(y)| & <\operatorname{diam}(f(D))=\operatorname{diam}(f(E)) \leq 2 \max _{z \in E}|f(x)-f(z)| \\
& \leq 2 \max _{z \in E} \omega(|x-z|) \leq 2 \omega(|x-y|)
\end{aligned}
$$

for all $y \in S(x, r)$.

In the remaining case, $f(x)$ lies in the bounded component of $\mathbf{R}^{n} \backslash f(S(x, r))$. Let $z$ be a point in $\partial D \backslash B(x, r)$ closest to $x$. Fix $R>r$ satisfying

$$
\frac{1}{2}|x-z| \leq b R \leq|x-z| .
$$

By Lemmas 1 and 2 in $[\mathrm{MN}]$, there is a constant $c \geq 1$, depending only on $a, b, n$ and $K_{O}(f)$, such that

$$
|f(x)-f(y)| \leq L(r) \leq c l(r) \leq c L(R)\left(\frac{r}{R}\right)^{\alpha} \leq c^{2} l(R)\left(\frac{r}{R}\right)^{\alpha}
$$

for all $y \in S(x, r)$. Since

$$
l(R) \leq|f(x)-f(z)| \leq \omega(|x-z|) \leq \omega(2 b R) \leq 4 b \omega(R),
$$

we thereby obtain

$$
|f(x)-f(y)| \leq 4 b c^{2} \frac{\omega(R)}{R^{\alpha}}|x-y|^{\alpha} .
$$

Now, because either (2.7) or (2.8) must be true for each $y \in S(x, r)$, inequality (2.5) follows with $C=\max \left\{2,4 b c^{2} M\right\}$.

Finally, if $\omega(t) / t^{\alpha}$ is decreasing, then

$$
\frac{\omega(R)}{R^{\alpha}}|x-y|^{\alpha} \leq \omega(|x-y|)
$$

in (2.8). As a combination of (2.7) and (2.8) this establishes (2.6) with $\hat{C}=4 b c^{2}$.

Lemma 2.5. Let $y$ be a point in a bounded domain $D$, let $x$ be a point in $\partial D$ closest to $y$, set $d=|x-y|$ and let

$$
\operatorname{cap}(\bar{B}(y, d / 2), D)<2^{-(n+3)} b_{n} .
$$

Suppose that $f$ is a continuous mapping of $\bar{D}$ into $\mathbf{R}^{n}$ which is quasiconformal in $D$ and that

$$
|f(x)-f(z)| \leq \omega(|x-z|)
$$

for all $z \in \partial D$, where $\omega$ is a majorant satisfying

$$
\frac{\omega(t)}{t^{\alpha}} \leq M \max \left\{1, \frac{\omega(s)}{s^{\alpha}}\right\}
$$

for some $M>0$, for $\alpha=K_{I}(f)^{1 /(1-n)}$ and for all $0<s<t<\operatorname{diam}(D)$. Then

$$
|f(x)-f(y)| \leq C \max \left\{\omega(|x-y|),|x-y|^{\alpha}\right\},
$$

where $C$ depends only on $n, M$ and $K_{O}(f)$. In particular, if $\omega(t) / t^{\alpha}$ is decreasing for $0<t<\operatorname{diam}(D)$, then

$$
|f(x)-f(y)| \leq \hat{C} \omega(|x-y|),
$$

where $\hat{C}$ depends only on $n$ and $K_{O}(f)$. 
Proof. Let $E$ denote the boundary component of $D$ whose image under $f$ separates $f(D)$ from the point $\infty$ in $\overline{\mathbf{R}}^{n}$. We distinguish two cases.

Suppose first that $E \subset B(x, 4 d)$. Then

$$
\begin{aligned}
|f(x)-f(y)| & \leq \operatorname{diam}(f(D))=\operatorname{diam}(f(E)) \leq \omega(\operatorname{diam}(E)) \\
& \leq \omega(8 d) \leq 8 \omega(d)=8 \omega(|x-y|) .
\end{aligned}
$$

Assume next that $E$ does not lie in $B(x, 4 d)$. Then, by Lemma 2.3, there is $r, d<r<2 d$, such that $S(x, r)$ is contained in $D$ and

$$
\frac{L(r)}{l(r)} \leq c,
$$

where $c \geq 1$ depends only on $n$ and $K_{O}(f)$. Pick a point $z$ in $\partial D \backslash B(x, 4 d)$ closest to $x$. Since $f(x)$ must lie in the bounded component of $\mathbf{R}^{n} \backslash f(S(x, r))$, we obtain

$$
|f(x)-f(y)| \leq L(r) \leq c l(r) \leq c|f(x)-f(z)| \leq c \omega(|x-z|) .
$$

Now if $|x-z| \leq 6|x-y|$, then

$$
|f(x)-f(y)| \leq c \omega(6|x-y|) \leq 8 c \omega(|x-y|),
$$

while if $|x-z|>6|x-y|$, then $D$ contains the closed ring $\bar{B}(x, 6 d) \backslash B(x, 4 d)$, and therefore, in view of Lemma 2.4,

$$
|f(x)-f(w)| \leq C^{\prime} \max \left\{\omega(|x-w|),|x-w|^{\alpha}\right\}
$$

for all $w \in S(x, 5 d)$, where $C^{\prime}>0$ depends only on $n, M$ and $K_{O}(f)$.

On the other hand, since the $f(x)$-component of $\mathbf{R}^{n} \backslash f(S(x, 5 d))$ is bounded, we infer that

$$
|f(x)-f(y)| \leq L(5 d) \leq C^{\prime} \hat{\omega}(5 d) \leq 8 C^{\prime} \hat{\omega}(d)=8 C^{\prime} \hat{\omega}(|x-y|),
$$

where $\hat{\omega}(t)=\max \left\{\omega(t), t^{\alpha}\right\}$. Therefore, because one of the inequalities (2.11), (2.12) and (2.14) must be true for $x$ and $y$, the conclusion (2.9) is secured with $C=$ $8 \max \left\{c, C^{\prime}\right\}$, a constant depending only on $n, M$ and $K_{O}(f)$.

Finally, if $\omega(t) / t^{\alpha}$ is decreasing, Lemma 2.4 allows us to improve inequality (2.13) to

$$
|f(x)-f(w)| \leq \tilde{C} \omega(|x-w|),
$$

which is valid for all $w \in S(x, 5 d)$ and wherein $\tilde{C}>0$ depends only on $n$ and $K_{O}(f)$. Consequently, (2.14) will transform into

$$
|f(x)-f(y)| \leq 8 \tilde{C} \omega(|x-y|) .
$$

Since one of the inequalities (2.11), (2.12) and (2.15) must be true for $x$ and $y$, the desired inference $(2.10)$ results with $\hat{C}=8 \max \{c, \tilde{C}\}$.

One last piece of preliminaries is needed in Section 3, and that is the concept of uniform perfectness introduced by Pommerenke in $[\mathrm{P}]$. A compact set $E$ in $\mathbf{R}^{n}$ is called $c$-uniformly perfect, $0<c<1$, if $E$ contains at least two points and if for each $x \in E$ and $0<r<\operatorname{diam}(E)$, the spherical ring $B(x, r) \backslash \bar{B}(x, c r)$ meets $E$. Such an $E$ is quite evidently a perfect set. For background information on uniformly perfect sets see, for example, [BP], [JV], [P].

There exist several alternative characterizations for uniformly perfect sets in the literature. We need the following result involving condition (2.1) in Lemma 2.2, see [AMV]: 
Lemma 2.6. If the boundary of a bounded domain $D$ in $\mathbf{R}^{n}$ is c-uniformly perfect then there exists $m>0$ such that

$$
\operatorname{cap}\left(\bar{B}\left(y, \frac{1}{2} d\right), D\right) \geq m
$$

for each point $y \in D$, where $d=d(y, \partial D)$. Here $m$ depends only on $c$ and on the dimension $n$.

Proof. It follows from Lemma 2.10 in [JV] that there is a constant $s, 0<s<\infty$, depending only on $c$ and $n$, such that $\bmod (R)<s$ for each ring domain $R$ which separates $\partial D$. The proof of Lemma 2.3 in [AMV] then implies that the assertion in Lemma 2.6 holds with a constant $m>0$ depending only on $c$ and $n$.

\section{Results}

We are now prepared to establish our main results. The first of these is a generalization of [NP, Theorem 1], due to Palka and one of the authors. See also [HN, Theorem 2]. We remind the reader that the number $\lambda_{n}$ in the ensuing theorems designates the familiar positive constant present in the Teichmüller ring domain estimate: if $E=\left\{t e_{1}:-1 \leq t \leq 0\right\}$ and $F_{t}=\left\{s e_{1}: t \leq s<\infty\right\}$ for $t>0$, then we have

$$
M\left(\Delta\left(E, F_{t} ; \mathbf{R}^{n}\right)\right) \geq \frac{\omega_{n-1}}{\left[\log \lambda_{n}(t+1)\right]^{n-1}},
$$

see $[\mathrm{AF}],[\mathrm{AVV}],[\mathrm{G}]$. The exact value of $\lambda_{n}$ is somewhat mysterious, except when $n=2$, in which event $\lambda_{2}=16$. The best bounds for $\lambda_{n}$ known to us, when $n>2$, are

$$
4 e^{1,52(n-1)}<\lambda_{n}<4 e^{2(n-1)} .
$$

Theorem 3.1. Let $D$ be a proper subdomain of $\mathbf{R}^{n}$ and let $f$ be a continuous mapping of $\bar{D}$ into $\mathbf{R}^{n}$ which is quasiconformal in $D$. Suppose that

$$
|f(x)-f(y)| \leq \omega(|x-y|)
$$

for some majorant $\omega$ and for all $x \in \partial D$ and $y \in D$. Then

$$
|f(x)-f(y)| \leq C \max \left\{\omega(|x-y|), \frac{\omega(d)}{d^{\alpha}}|x-y|^{\alpha}\right\}
$$

for all $x, y \in D$, where $\alpha=K_{I}(f)^{1 /(1-n)}, d=d(y, \partial D)$ and $C=4 \lambda_{n}$, a constant depending only on $n$ and satisfying $16 e^{1,52(n-1)}<C<16 e^{2(n-1)}$. In particular, if $\omega(t) / t^{\alpha}$ is decreasing for $0<t<\operatorname{diam}(D)$, then

$$
|f(x)-f(y)| \leq C \omega(|x-y|)
$$

for all $x, y \in D$, where again $C=4 \lambda_{n}$.

Proof. The argument here closely mimics the one used in [NP, p. 380] for a Hölder continuous function $\omega$.

Fix distinct points $x$ and $y$ in $D$ and select a point $z \in \partial D$ closest to $y$. If $|z-y| \leq|x-y|$, then $|z-x| \leq 2|x-y|$, and (3.1) yields

$$
|f(x)-f(y)| \leq 3 \omega(|x-y|) .
$$

Assume next that $|z-y|>|x-y|$ and set

$$
d=|z-y|, r=|x-y|
$$


The spherical ring domain $R=B(y, d) \backslash \bar{B}(y, r)$ lies in $D$. The curve family $\Gamma$ in $R$ joining the boundary components of $R$ has modulus

$$
M(\Gamma)=\frac{\omega_{n-1}}{\left(\log \frac{d}{r}\right)^{n-1}} .
$$

As in [NP, p. 380], one can use [G, Theorem 4] together with the Teichmüller ring domain estimate to derive

$$
M(f \Gamma) \geq \omega_{n-1}\left[\log \lambda_{n}\left(1+\frac{|f(z)-f(y)|}{|f(x)-f(y)|}\right)\right]^{1-n} .
$$

Since $|z-x|<2 d$, it follows from (3.1) and the triangle inequality that

$$
|f(x)-f(y)| \leq|f(x)-f(z)|+|f(z)-f(y)| \leq 3 \omega(d) .
$$

This, in conjunction with (3.1) and (3.6), gives

$$
M(f \Gamma) \geq \omega_{n-1}\left[\log \lambda_{n} \frac{4 \omega(d)}{|f(x)-f(y)|}\right]^{1-n} .
$$

Now $M(f \Gamma) \leq K_{I}(f) M(\Gamma)$ by the quasiconformality of $f$ and we infer, combining (3.5) and (3.7), that

$$
|f(x)-f(y)| \leq \frac{4 \lambda_{n} \omega(d) r^{\alpha}}{d^{\alpha}}=\frac{4 \lambda_{n} \omega(d)}{d^{\alpha}}|x-y|^{\alpha} .
$$

Consequently, because either (3.4) or (3.8) must hold for each pair of points $x$ and $y$ in $D$, inequality (3.2) will follow with $C=4 \lambda_{n}$.

Finally, if $\omega(t) / t^{\alpha}$ is decreasing, then

$$
\frac{\omega(d)}{d^{\alpha}}|x-y|^{\alpha} \leq \omega(|x-y|)
$$

in (3.8). Since either (3.4) or (3.8) must hold for any pair of points $x, y \in D$, inequality (3.3) is verified with $C=4 \lambda_{n}$.

In dimension $n=2$, the dilatations $K_{I}(f), K_{O}(f)$ and $K(f)$ are all equal and the constant $C$ in Theorem 3.1 is nothing but 64 . Hence we may record the following consequence:

Corollary 1. Under the hypotheses of Theorem 3.1, if $n=2$, then

$$
|f(x)-f(y)| \leq 64 \max \left\{\omega(|x-y|), \frac{\omega(d)}{d^{1 / K}}|x-y|^{1 / K}\right\}
$$

for all $x, y \in D$, where $d=d(y, \partial D)$ and $K$ is the dilatation of the quasiconformal mapping $f$. In particular, if $\omega(t) / t^{1 / K}$ is decreasing when $0<t<\operatorname{diam}(D)$, then

$$
|f(x)-f(y)| \leq 64 \omega(|x-y|)
$$

for all $x, y \in D$.

Remark 1. In Theorem 3.1, condition (3.1) by itself affords no guarantee that the estimate (3.3) be satisfied for any fixed constant $C$, even if $D$ were assumed to be a bounded domain with smooth boundary. For example, the radial stretching $f$,

$$
f(x)=|x|^{\alpha-1} x, 0<\alpha<1,
$$

carries the unit ball $B$ in $\mathbf{R}^{n}$ onto itself, is the identity in $\partial B$, and is quasiconformal in $B$ with $K_{I}(f)=\alpha^{1-n}$. See $[\mathrm{V}, 16.2]$. Moreover, for points $x \in \partial B$ and $y \in B$ we 
have $|f(x)-f(y)|<2|x-y|$, and so (3.1) is fulfilled with $\omega(t)=2 t$. However, since $f$ is Hölder continuous at the origin with optimal Hölder exponent $\alpha$, the estimate (3.3) in Theorem 3.1 cannot possibly be achieved for any fixed constant $C$.

Hinkkanen and one of the present co-authors established the following result in [HN, Theorem 4]: if $B$ is the open unit ball in $\mathbf{R}^{n}$ and if $f$ is a continuous mapping of $\bar{B}$ into $\mathbf{R}^{n}$ which is quasiconformal in $B$ and satisfies (3.1) for all $x, y \in \partial B$ and for some majorant $\omega$, then

$$
|f(x)-f(y)| \leq C \omega(|x-y|)
$$

for all $x \in \partial B, y \in B$, where $C>0$ depends only on $n$ and $K(f)$. The upshot of this is that should the domain $D$ in Theorem 3.1 be a ball, it would suffice to assume (3.1) for points $x, y \in \partial D$ only, when deriving (3.2) and (3.3), at the expense of a possible dependence on the dilation $K(f)$ for the constant $C$. The realm of domains in which a similar derivation is valid is, in fact, substantially larger, as will next be demonstrated:

Theorem 3.2. Let $D$ be a bounded domain in $\mathbf{R}^{n}$ and let $\partial D$ be c-uniformly perfect. If $f$ is a continuous mapping of $\bar{D}$ into $\mathbf{R}^{n}$ which is quasiconformal in $D$ and if

$$
|f(x)-f(y)| \leq \omega(|x-y|)
$$

for all $x, y \in \partial D$ and for some majorant $\omega$, then

$$
|f(x)-f(y)| \leq C \omega(|x-y|)
$$

for all $x \in \partial D$ and $y \in D$, where $C$ depends only on $c, n, K(f)$ and $\operatorname{diam}(D)$. Furthermore,

$$
|f(x)-f(y)| \leq \hat{C} \max \left\{\omega(|x-y|), \frac{\omega(d)}{d^{\alpha}}|x-y|^{\alpha}\right\}
$$

for all $x, y \in D$, where $\hat{C}=4 \lambda_{n} C, d=d(y, \partial D)$ and $\alpha=K_{I}(f)^{1 /(1-n)}$. In particular, if $\omega(t) / t^{\alpha}$ is decreasing for $0<t<\operatorname{diam}(D)$, then

$$
\mid f(x)-f(y) \leq \hat{C} \omega(|x-y|)
$$

for all $x, y \in D$, where $\hat{C}$ is as above.

Proof. Since $D$ is bounded and $\partial D$ is $c$-uniformly perfect, Lemma 2.6 will provide us a constant $m>0$, which depends only on $n, c$ and $\operatorname{diam}(D)$, such that

$$
\operatorname{cap}\left(\bar{B}\left(y, \frac{1}{2} d\right), D\right) \geq m
$$

for each point $y \in D$, where $d=d(y, \partial D)$. Invoking Lemma 2.2 we thus obtain another constant $C_{1}$, depending only on $n, m, K(f)$ and diam $(D)$, with the property that

$$
|f(x)-f(y)| \leq C_{1} \omega(|x-y|)
$$

for each pair of points $x \in \partial D$ and $y \in D$ such that $|x-y|=d(y, \partial D)$. The conclusion (3.10) ensues, therefore, by virtue of Lemma 2.1, for all points $x \in \partial D$ and $y \in D$ with $C=3 C_{1}$. The estimate (3.11) in Theorem 3.2 is now achieved by combining (3.10) with Theorem 3.1. The last assertion is a special case of Theorem 3.1. 
Remark 2. Condition (3.9) for all $x, y \in \partial D$, without any assumption of the type that $\partial D$ be uniformly perfect or that $\partial D$ have positive local capacity, is not enough to imply that (3.10) be valid for all $x \in \partial D$ and $y \in D$. For example, if $D$ is the punctured unit ball $B \backslash\{0\}$ in $\mathbf{R}^{n}$ and if $f$ is the radial stretching defined in Remark 1, then (3.9) obviously holds for all $x, y \in \partial D$ with $\omega(t)=t$. But selecting $x=0$ we see that no fixed $C$ can ensure the estimate (3.10) for all $y \in D$.

We conclude this paper with our main result. It will furnish an extension promised in the introduction of the theorem in [MN] involving Hölder continuous boundary majorants.

Theorem 3.3. Let $D$ be a bounded domain in $\mathbf{R}^{n}$ and let $f$ be a continuous mapping of $\bar{D}$ into $\mathbf{R}^{n}$ which is quasiconformal in $D$. Suppose that

$$
|f(x)-f(y)| \leq \omega(|x-y|)
$$

for all $x, y \in \partial D$, where $\omega$ is a majorant satisfying

$$
\frac{\omega(t)}{t^{\alpha}} \leq M \max \left\{1, \frac{\omega(s)}{s^{\alpha}}\right\}
$$

for some $M>0$, for $\alpha=K_{I}(f)^{1 /(1-n)}$ and for all $0<s<t<\operatorname{diam}(D)$. Then

$$
|f(x)-f(y)| \leq C \max \left\{\omega(|x-y|),|x-y|^{\alpha}\right\}
$$

for all $x, y \in D$, where $C$ depends only on $n, M$ and $K(f)$. In particular, if $\omega(t) / t^{\alpha}$ is decreasing for $0<t<\operatorname{diam}(D)$, then

$$
|f(x)-f(y)| \leq \hat{C} \omega(|x-y|)
$$

for all $x, y \in D$, where $\hat{C}$ depends only on $n$ and $K(f)$.

Proof. Let $y \in D$, let $z$ be a point in $\partial D$ closest to $y$ and set $d=|y-z|$. If

$$
\operatorname{cap}\left(\bar{B}\left(y, \frac{1}{2} d\right), D\right) \geq 2^{-(n+3)} b_{n}
$$

then, by Lemma 2.2, there is a constant $C_{1}>0$, depending only on $n$ and $K(f)$, such that

$$
|f(z)-f(y)| \leq C_{1} \omega(|z-y|) .
$$

If (3.15) fails, then, by Lemma 2.5, there is a constant $C_{2}>0$, depending only on $n, M$ and $K(f)$, such that

$$
|f(z)-f(y)| \leq C_{2} \max \left\{\omega(|z-y|),|z-y|^{\alpha}\right\} .
$$

Consequently,

$$
|f(z)-f(y)| \leq C_{3} \hat{\omega}(|z-y|)
$$

whenever $y \in D, z$ is a point in $\partial D$ closest to $y, C_{3}=\max \left\{C_{1}, C_{2}\right\}$, and $\hat{\omega}(t)=$ $\max \left\{\omega(t), t^{\alpha}\right\}$.

Now $\hat{\omega}$ is also a majorant. Lemma 2.1 thereby implies that

$$
|f(x)-f(y)| \leq 3 C_{3} \hat{\omega}(|x-y|)
$$


for all $x \in \partial D$ and $y \in D$. Theorem 3.1 in turn yields

$$
\begin{aligned}
|f(x)-f(y)| & \leq 12 \lambda_{n} C_{3} \max \left\{\hat{\omega}(|x-y|), \frac{\hat{\omega}(d)}{d^{\alpha}}|x-y|^{\alpha}\right\} \\
& =12 \lambda_{n} C_{3} \max \left\{\omega(|x-y|),|x-y|^{\alpha}, \frac{\omega(d)}{d^{\alpha}} \mid x-y^{\alpha}\right\}
\end{aligned}
$$

for all $x, y \in D$, where $d=d(y, \partial D)$. This combined with our hypothesis (3.12) establishes (3.13) with $C=12 \lambda_{n} M C_{3}$.

Finally, suppose that $\omega(t) / t^{\alpha}$ is decreasing. Lemma 2.5 enables us to improve inequality (3.17) to read

$$
|f(z)-f(y)| \leq C_{2} \omega(|z-y|)
$$

where $C_{2}$ now depends only on $n$ and $K(f)$. This, together with (3.16), allows one to infer that

$$
|f(z)-f(y)| \leq C_{3} \omega(|z-y|)
$$

whenever $y \in D, z$ is a point in $\partial D$ closest to $y$ and $C_{3}=\max \left\{C_{1}, C_{2}\right\}$. While Lemma 2.1 guarantees that

$$
|f(x)-f(y)| \leq 3 C_{3} \omega(|x-y|)
$$

for all $x \in \partial D$ and $y \in D$, Theorem 3.1 then will deliver the desired estimate (3.14) with $\hat{C}=12 \lambda_{n} C_{3}$, a constant depending only on $n$ and $K(f)$.

Remark 3. If, in Theorem 3.3, $n=2$ and if the mapping $f$ is conformal, or merely analytic, then a myriad of results are known that are much more complete and satisfactory than ours. See, for example, [GHH], [H2], [H3], [RST], [S], [T]. The most striking results are due to Hinkkanen. In [H3] he showed that if $D$ is a bounded domain in the complex plane $\mathbf{C}$ and if $f$ is a continuous mapping of $\bar{D}$ into $\mathbf{C}$ which is analytic in $D$ and satisfies $|f(x)-f(y)| \leq \omega(|x-y|)$ for some majorant $\omega$ and for all $x, y \in \partial D$, then $|f(x)-f(y)| \leq C \omega(|x-y|)$ for all $x, y \in D$, where $C=3456$. Furthermore, if $D$ happens to be the unit disk, Hinkkanen [H2] showed that the constant above can be reduced to $C=2$. It follows from results of Hinkkanen [H2], [H3], and of Smith and Stegenga [SS] that the constant 2 above is the best possible in the case where only one of the points $x$ and $y$ above is allowed to vary. That the tantalizing number 3456 were to enjoy any such sharpness property seems rather unlikely. For Hölder continuous majorants the best constant above is $C=1$, as was probably first shown by Sewell [S] and later on by Rubel, Shields and Taylor [RST] in the case of a disk, and by Gehring, Hayman and Hinkkanen [GHH] in the case of an arbitrary bounded domain $D$.

\section{References}

[AF] Anderson, G. D., and J. S. Frame: Numerical estimates for a Grötzsch ring constant. Constr. Approx. 4, 1988, 223-242.

[AVV] Anderson, G. D., M. K. Vamanamurthy, and M. Vuorinen: Conformal invariants, inequalities, and quasiconformal maps. - Wiley, New York, 1997.

[AMV] Arsenović, M., V. Manojlović, and M. Vuorinen: Hölder continuity of harmonic quasiconformal mappings. - J. Inequal. Appl. 2011:37, 2011.

[BP] Beardon, A.F., and Ch. Pommerenke: The Poincaré metric of plane domains. - J. London Math. Soc. 18, 1978, 475-483. 
[G] Gehring, F. W.: Symmetrizations of rings in space. - Trans. Amer. Math. Soc. 103, 1962, 353-393.

[GHH] Gehring, F. W., W. K. Hayman, and A. Hinkkanen: Analytic functions satisfying Hölder conditions on the boundary. - J. Approx. Theory 35, 1982, 243-249.

[H1] Hinkkanen, A.: Modulus of continuity of harmonic functions. - J. Anal. Math. 50, 1988, $1-29$.

[H2] Hinkkanen, A.: The sharp form of certain majorization theorems for analytic functions. - Complex Var. Theory Appl. 12, 1989, 39-66.

[H3] Hinkkanen, A.: Majorization of the modulus of continuity of analytic functions. - Comput. Methods Funct. Theory 8, 2008, 303-325.

[HN] HinkKanen, A., and R. NÄKkI: Analytic functions and quasiconformal mappings in Stolz angles and cones. - Complex Var. Theory Appl. 13, 1990, 251-267.

[JV] JÄRVI, P., and M. VUORINEn: Uniformly perfect sets and quasiregular mappings. - J. London Math. Soc. (2) 54, 1996, 515-529.

[K] KalaJ, D.: On quasiregular mappings between smooth Jordan domains. - J. Math. Anal. Appl. 362, 2010, 58-63.

[KM] Kalaj, D., and M. Mateljević: Inner estimate and quasiconformal harmonic mappings between smooth domains. - J. Anal. Math. 100, 2006, 117-132.

[MN] Martio, O., and R. NÄKKI: Boundary Hölder continuity and quasiconformal mappings. J. London Math. Soc. (2) 44, 1991, 339-350.

[NP] NÄKKI, R., and B. PALKA: Lipschitz conditions and quasiconformal mappings. - Indiana Univ. Math. J. 31:3, 1982, 377-401.

[P] Pommerenke, Ch.: Uniformly perfect sets and the Poincaré metric. - Arch. Math. 32, 1979, 192-199.

[RST] Rubel, L. A., A. L. Shields, and B. A. TAYlor: Mergelyan sets and the modulus of continuity of analytic functions. - J. Approx. Theory 15, 1975, 23-40.

[S] Sewell, W. E.: Degree of approximation by polynomials in the complex domain. - Ann. of Math. Stud. 9, Princeton Univ. Press, Princeton, N.J., 1942.

[SS] Smith, W., and D. Stegenga: The local modulus of continuity of an analytic function. - In: Holomorphic functions and moduli, Vol. I, Math. Sci. Res. Inst. Publ. 10, Springer, New York, 1988, 133-142.

[T] Tamrazov, P. M.: Contour and solid structure properties of holomorphic functions of a complex variable. - Russian Math. Surveys 28, 1973, 141-173.

[V] VÄISÄLÄ, J.: Lectures on $n$-dimensional quasiconformal mappings. - Lecture Notes in Math. 229, Springer-Verlag, Berlin/Heidelberg/New York, 1971.

Received 17 December 2010

Revised received 10 October 2011 